\title{
Análisis de la mortalidad perinatal en Lima Norte: uso del método BABIES
}

Luz E. Chan 1,a ; Lily G. Reyes ${ }^{1, a}$; Judith Cahuana-Aparco 1,2,b; Paul Jesús Tejada-Llacsa* 2,b

RESUMEN

Objetivo: Identificar la "Brecha de Oportunidad" mediante el método BABIES con el empleo de tasas de mortalidad perinatal.

Materiales y métodos: Se realizó un estudio descriptivo transversal con la Base de datos de Vigilancia Epidemiológica Perinatal y Neonatal. Se determinó las tasas de mortalidad perinatal por distritos y por áreas de posible intervención según método BABIES.

Resultados: La tasa de mortalidad más baja se encontró en el distrito de Independencia (6,1 por 100 nacimientos vivos en 2014, y 5,9 en 2015, respectivamente). El área prioritaria de intervención en el distrito de Comas fue salud materna, y en Carabayllo, Cuidado durante el embarazo.

Conclusiones: El método BABIES permite identificar la Brecha de Oportunidad para poder dirigir intervenciones a nivel local.

Palabras clave: Mortalidad perinatal; Salud materna; Vigilancia (Fuente: DeCS BIREME).

\section{Analysis of perinatal mortality in Northern Lima: using the BABIES method}

\section{ABSTRACT}

Objective: To identify the "opportunity gap" with the BABIES method using perinatal mortality rates.

Materials and methods: A descriptive cross-sectional study was performed with the Perinatal and Neonatal Epidemiological Surveillance Database. Perinatal mortality rates by districts and by areas of possible intervention were determined according to the BABIES method.

Results: The district of Independencia had the lowest mortality rate, with 6.1 and 5.9 per 100 live births in the years 2014 and 2015, respectively. The priority area of intervention in the district of Comas was "maternal health" and in Carabayllo, "care during pregnancy".

Conclusions: The BABIES method allows us to identify the "opportunity gap" which can guide us to perform interventions at the local level.

Keywords: Perinatal mortality; Maternal health; Surveillance (Source: MeSH NLM).

1. Red de Salud Túpac Amaru. Lima, Perú.

2. Asociación para el Desarrollo de la Investigación Estudiantil en Ciencias de la Salud (ADIECS-UNMSM). Lima, Perú.

a. Médico Salubrista.

b. Médico Cirujano.

* Autor corresponsal. 


\section{INTRODUCCIÓN}

La mortalidad perinatal representa condiciones perinatales inadecuadas de los países en vías de desarrollo. La Organización Mundial de la Salud estimó que el 98 \% de las muertes perinatales ocurren en países en vías de desarrollo ${ }^{(1)}$. En el Perú, la mortalidad perinatal es de 14 defunciones por mil embarazos para el año 2017, según la Encuesta Nacional Demográfica y de Salud Familiar (2). La tasa de mortalidad perinatal engloba las muertes fetales y neonatales; debido a esto, sus valores permiten tener una visión de la atención prenatal, durante el parto y posparto. Además, representa un problema de salud prioritario a nivel mundial y nacional (3) por lo que es necesario tomar acciones en distintos niveles dirigidas a la gestante y recién nacidos. Los decisores de salud juegan un papel clave para la toma de decisiones e impulso de intervenciones, por lo cual resulta necesario contar con herramientas que permitan realizar un análisis local del problema y poder dirigir esfuerzos.

La Norma Técnica de Salud que establece el Subsistema Nacional de Vigilancia Epidemiológica Perinatal y Neonatal (4), aprobada en abril del 2009 , señala que el análisis de la información en todos los niveles del sistema se realizará con el método BABIES (Birth Weight and Age-at-death Boxes for an Intervention and Evaluation System) ${ }^{(5-8)}$.

Esta es una herramienta de evaluación adaptable que utiliza dos variables, edad en el momento de la muerte del feto-recién nacido y peso al nacer, lo cual permite analizar estos datos para identificar la Brecha de Oportunidad y con ello, priorizar intervenciones para reducir la muerte perinatal. La "brecha de oportunidad" es la diferencia entre las tasas de mortalidad de una subpoblación que ha logrado un mejor estado de salud con aquellas experimentadas por la población restante. Existe una "oportunidad" para lograr la equidad entre las poblaciones al mejorar la disponibilidad, accesibilidad, aceptabilidad, asequibilidad y la adecuación de los servicios para reducir la "brecha" (5). Existen reportes del uso y utilidad del método BABIES en Tanzania ${ }^{(6)}$ y Estados Unidos ${ }^{(8)}$.

Actualmente, se realiza un reporte nacional y local donde se estima el porcentaje relativo a todas las muertes perinatales de acuerdo a 4 áreas (Salud materna, Cuidado durante el embarazo, Cuidado durante el parto y Cuidado del recién nacido). Sin embargo, no existen reportes de la identificación de la Brecha de Oportunidad, y es importante calcularla, ya que por cada área identificada existe un paquete de posibles intervenciones en Salud Pública ${ }^{(5)}$. Por tal motivo, nuestro objetivo es identificar la Brecha de Oportunidad mediante el método BABIES; y el objetivo secundario es describir las características de la mortalidad perinatal de los distritos de Independencia, Comas y Carabayllo en la Red de Salud Túpac Amaru.

\section{MATERIALES Y MÉTODOS}

\section{Diseño y población}

El diseño de estudio fue descriptivo transversal. Se realizó el análisis secundario de los casos de muerte perinatal registrados desde el 1 de enero del 2014 hasta el 31 de diciembre del 2015 en el Subsistema de Vigilancia Epidemiológica Perinatal y Neonatal (SSVEPN) del área de epidemiología de la Red de Salud Túpac Amaru.

La unidad de análisis fue el caso de muerte perinatal reportado en los establecimientos de salud de los distritos de Independencia, Comas y Carabayllo. El registro de los casos fue realizado por los responsables de epidemiología de los establecimientos de salud en la ficha de notificación de muertes perinatales. Luego se digitalizó la información en el aplicativo NotiWebversión 2.5.

\section{Variables y mediciones}

Las variables consideradas fueron año, sexo, edad gestacional, fecha de nacimiento, fecha de fallecimiento, hora de nacimiento, hora de muerte, peso de nacimiento, tipo de muerte (categorizado como fetal o neonatal) y la categoría de la causa básica de muerte.

La categoría de la causa básica de muerte se consideró de acuerdo al CIE-10: P00 (Feto y recién nacido afectados por condiciones de la madre no necesariamente relacionadas con el embarazo presente), P02 (Feto y recién nacido afectados por complicaciones de la placenta, del cordón umbilical y de las membranas), P07 (Trastornos relacionados con duración corta de la gestación y con bajo peso al nacer, no clasificados en otra parte), P21 (Asfixia del nacimiento), P28 (Otros problemas respiratorios del recién nacido, originados en el período perinatal), P36 (Sepsis bacteriana del recién nacido), P95 (Muerte fetal de causa no especificada), Q00-Q99 (Malformaciones congénitas, deformidades y anomalías cromosómicas), las demás categorías de diagnóstico se agruparon como "Otras".

Para la identificación de la Brecha de Oportunidad se utilizó el método BABIES, el cual evalúa la distribución de las muertes perinatales en una tabla de doble entrada; las variables son el peso al nacer y la edad al momento de la muerte. La distribución de las celdas se agrupan en 4 áreas que son Salud materna, Cuidado durante el embarazo, Cuidado durante el parto y Cuidado del recién nacido (Figura 1). Luego se calcula las tasas de mortalidad perinatal por cada área. 


\begin{tabular}{|c|c|c|c|c|c|}
\hline \multirow{2}{*}{$\begin{array}{c}\text { PESO } \\
\text { (GRAMOS) }\end{array}$} & \multicolumn{2}{|c|}{ Muerte fetal } & \multicolumn{3}{c|}{ Muerte neonatal } \\
\cline { 4 - 6 } & $\begin{array}{c}\text { Antes del } \\
\text { parto }\end{array}$ & Intraparto & \multicolumn{3}{|c|}{ Después del parto } \\
\cline { 4 - 6 } & & & $<24 \mathrm{~h}$ & $1-7$ días & $8-28$ días \\
\hline $500-1499$ & & & & & \\
\hline $1500-2499$ & & & & & \\
\hline 2500 a más & & & & & \\
\hline
\end{tabular}

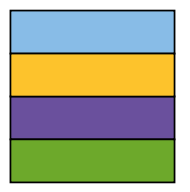

Salud materna

Cuidado durante el embarazo

Cuidado durante el parto

Cuidado del recién nacido

Figura 1. Distribución de casos de mortalidad perinatal según método BABIES

Para el cálculo del exceso de tasa de mortalidad perinatal se realiza la siguiente diferencia:

(Tasa de mortalidad perinatal calculada por cada área)(Tasa de mortalidad perinatal de la población estándar por cada área $)=($ Exceso de tasa de mortalidad perinatal $)$

La población estándar puede ser externa (tasa de mortalidad perinatal de un país desarrollado), nacional (tasa de mortalidad perinatal nacional), o interna (tasa de mortalidad perinatal dentro de una subpoblación de una región a analizar). De acuerdo al método BABIES, la más recomendado para un análisis local es la interna (en nuestro caso, la población estándar es el distrito de Independencia debido a su baja tasa de mortalidad perinatal).

Finalmente, esta diferencia, a la que se le denomina exceso de tasa, nos permite identificar la brecha de oportunidad y priorizar intervenciones en Salud Pública.

\section{Análisis estadístico}

Para calcular la tasa de mortalidad perinatal se colocó en el numerador los casos de mortalidad registrados en el sistema de vigilancia, y como denominador, el total de recién nacidos reportado por cada distrito según el Instituto Nacional de Estadística e Informática para el año 2014 y 2015. Luego se calcularon las tasas por cada área (Salud materna, Cuidado durante el embarazo, Cuidado durante el parto y Cuidado del recién nacido). Para la identificación de la Brecha de oportunidad se realizó el cálculo del exceso de tasa de mortalidad perinatal. Para ello, se consideró como población estándar de referencia al distrito con menor tasa de mortalidad perinatal en 2014 y 2015. Para la descripción de datos cuantitativos se usó la media y desviación estándar, los datos categóricos se expresaron en porcentajes. Se realizó un gráfico de barras para la distribución de muertes según la hora de nacimiento. Se utilizó STATA 14.

\section{Consideraciones éticas}

Se obtuvo el permiso para usar la base de datos por la Red de Salud Túpac Amaru.

\section{RESULTADOS}

Entre los años 2014 al 2015 se obtuvieron 317 registros de muertes perinatales. El sexo masculino presentó una frecuencia de 177 (55,8 \%). La edad gestacional media fue de $32,3 \pm 5,3$ semanas.

Se encontró que el mayor número de casos de muerte ocurrió por muerte fetal, excepto en el distrito de Independencia, donde fue por muerte neonatal. En relación a las causas de defunción, se observó que la más frecuente, en el total de casos, fue "feto y recién nacido afectado por condiciones de la madre"; sin embargo, esta proporción fue distinta en Independencia donde la principal causa fue "feto y recién nacido afectados por complicaciones de la placenta, del cordón umbilical y de las membranas" (Tabla 1). 
Tabla 1. Características de casos de mortalidad perinatal en los distritos de Comas, Carabayllo e Independencia durante el periodo 2014-2015

\begin{tabular}{|c|c|c|c|c|}
\hline \multirow[t]{2}{*}{ Caracteristicas } & \multicolumn{4}{|c|}{ Distrito } \\
\hline & $\begin{array}{c}\text { Comas } \\
\mathrm{n}(\%)\end{array}$ & $\begin{array}{l}\text { Carabayllo } \\
\text { n (\%) }\end{array}$ & $\begin{array}{l}\text { Independencia } \\
\text { n (\%) }\end{array}$ & Total \\
\hline \multicolumn{5}{|l|}{ Sexo } \\
\hline Masculino & $101(56,1)$ & $50(55,6)$ & $26(55,3)$ & $177(55,8)$ \\
\hline Femenino & $78(43,3)$ & $40(44,4)$ & $19(40,4)$ & $137(43,2)$ \\
\hline Indeterminado & $1(0,6)$ & 0 & $2(4,3)$ & $3(1,0)$ \\
\hline \multicolumn{5}{|l|}{ Edad gestacional en semanas } \\
\hline Menos de 28 & $43(23,9)$ & $18(20,0)$ & $13(27,7)$ & $74(23,4)$ \\
\hline 28 a 31 & $40(20,2)$ & $15(16,7)$ & $6(12,8)$ & $61(19,2)$ \\
\hline 32 a 36 & $50(27,8)$ & $30(33,3)$ & $12(25,5)$ & $92(29,0)$ \\
\hline 37 a 42 & $47(26,1)$ & $27(30,0)$ & $16(34,0)$ & $90(28,4)$ \\
\hline \multicolumn{5}{|l|}{ Peso en gramos } \\
\hline 500 a 1499 & $86(47,8)$ & $36(40,0)$ & $21(44,7)$ & $143(45,1)$ \\
\hline 1500 a 2499 & $40(22,2)$ & $23(25,6)$ & $7(14,9)$ & $70(22,1)$ \\
\hline 2500 a 3999 & $49(27,2)$ & $29(32,2)$ & $17(36,2)$ & $95(30,0)$ \\
\hline 4000 a más & $5(2,8)$ & $2(2,2)$ & $2(4,2)$ & $9(2,8)$ \\
\hline \multicolumn{5}{|l|}{ Tipo de muerte } \\
\hline Fetal & $95(52,8)$ & $51(56,7)$ & $23(48,9)$ & $169(53,3)$ \\
\hline Neonatal & $85(47,2)$ & $39(43,3)$ & $24(51,1)$ & $148(46,7)$ \\
\hline \multicolumn{5}{|l|}{ Año } \\
\hline 2014 & $87(48,3)$ & $52(57,8)$ & $24(51,1)$ & $163(51,4)$ \\
\hline 2015 & $93(51,7)$ & $38(42,2)$ & $23(48,9)$ & $154(48,6)$ \\
\hline \multicolumn{5}{|l|}{ Causa básica de muerte } \\
\hline $\begin{array}{l}\text { - Feto y recién nacido afectados por condiciones de la madre } \\
\text { no necesariamente relacionadas con el embarazo presente. }\end{array}$ & $52(28,9)$ & $26(28,9)$ & $2(4,26)$ & $80(25,2)$ \\
\hline $\begin{array}{l}\text { - Feto y recién nacido afectados por complicaciones de la } \\
\text { placenta, del cordón umbilical y de las membranas. }\end{array}$ & $9(5,0)$ & $8(8,9)$ & $12(25,5)$ & $29(9,2)$ \\
\hline $\begin{array}{l}\text { - Trastornos relacionados con duración corta de la gestación y } \\
\text { con bajo peso al nacer, no clasificados en otra parte. }\end{array}$ & $8(4,4)$ & $4(4,4)$ & $5(10,6)$ & $17(5,4)$ \\
\hline • Asfixia del nacimiento. & $3(1,7)$ & $1(1,1)$ & $1(2,1)$ & $5(1,6)$ \\
\hline $\begin{array}{l}\text { - Otros problemas respiratorios del recién nacido, originados en } \\
\text { el periodo perinatal. }\end{array}$ & $34(18,9)$ & $15(16,7)$ & $2(4,3)$ & $51(16,1)$ \\
\hline - Sepsis bacteriana del recién nacido. & $22(12,2)$ & $7(7,8)$ & $2(4,3)$ & $31(9,8)$ \\
\hline - Muerte fetal de causa no especificada. & $31(17,2)$ & $13(14,4)$ & $5(10,6)$ & $49(15,5)$ \\
\hline $\begin{array}{l}\text { - Malformaciones congénitas, deformidades y anomalías } \\
\text { cromosómicas. }\end{array}$ & $12(6,7)$ & $11(12,2)$ & $10(21,3)$ & $33(10,4)$ \\
\hline - Otras & $9(5,0)$ & $5(5,6)$ & $8(17,0)$ & $22(6,9)$ \\
\hline
\end{tabular}

La hora del día con mayor proporción de casos fue las 00:00 horas, con 64 nacimientos $(20,2 \%)$, esta tendencia se repitió en los tres distritos (Figura 2). 

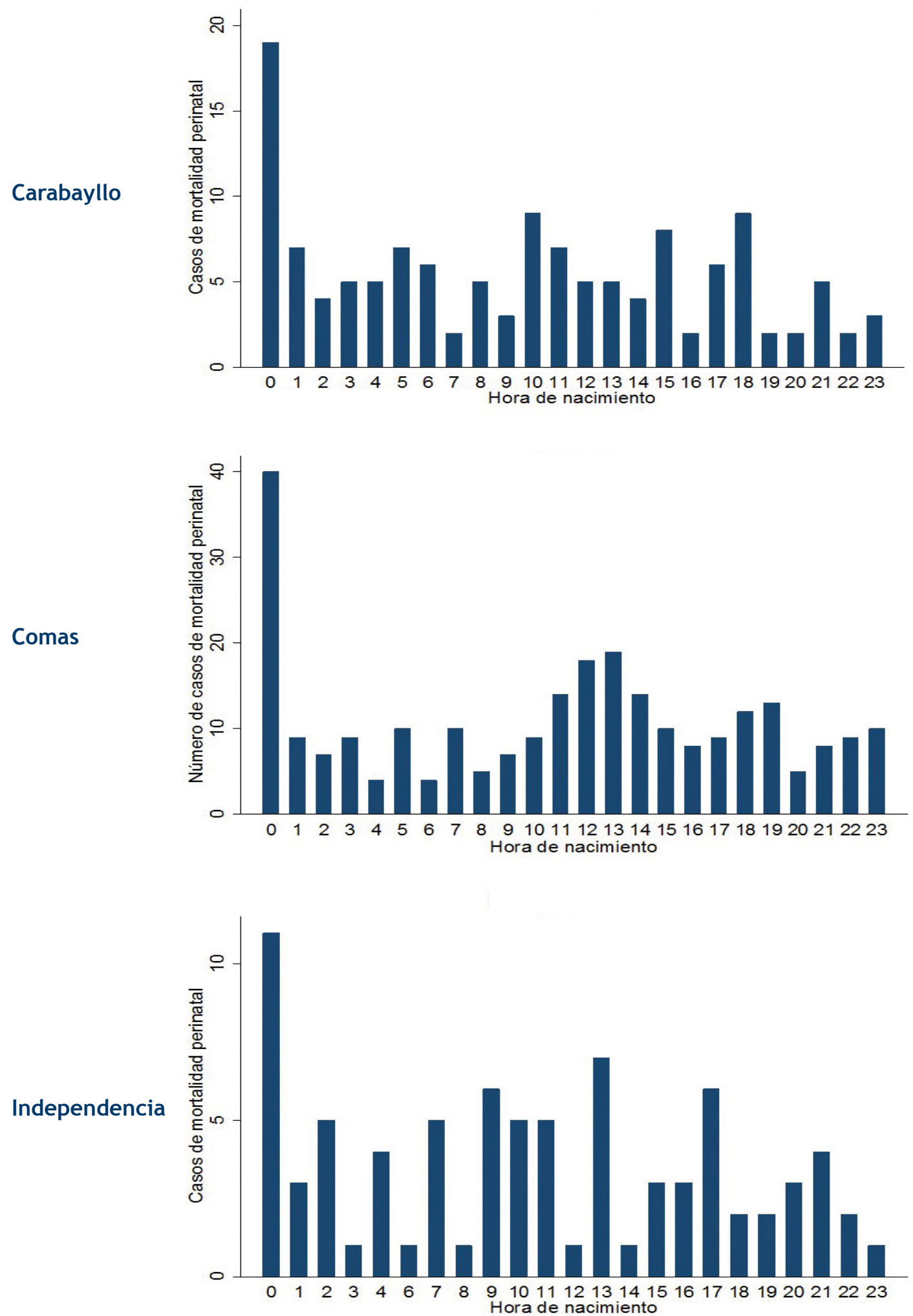

Figura 2. Número de casos de acuerdo a la hora de nacimiento en los distritos de Comas, Carabayllo e Independencia durante el periodo 2014-2015 
En relación a la identificación de la Brecha de Oportunidad, se obtuvo que en el distrito de Comas el exceso de mortalidad perinatal fue mayor en el área de
"Salud materna". Por otro lado, en Carabayllo el exceso fue mayor en el área de "Cuidado durante el embarazo" para los años 2014 y 2015 (Tabla 2).

Tabla 2. Identificación de exceso de tasa de mortalidad perinatal de acuerdo a metodología BABIES

\begin{tabular}{|c|c|c|c|c|c|}
\hline Distrito & $\begin{array}{l}\text { Salud } \\
\text { materna }\end{array}$ & $\begin{array}{l}\text { Cuidado } \\
\text { durante el } \\
\text { embarazo }\end{array}$ & $\begin{array}{c}\text { Cuidado } \\
\text { durante el } \\
\text { parto }\end{array}$ & $\begin{array}{l}\text { Cuidado } \\
\text { del recien } \\
\text { nacido }\end{array}$ & Total \\
\hline \multicolumn{6}{|l|}{ Independencia* } \\
\hline Número de muertes perinatales & 21 & 13 & 1 & 12 & 47 \\
\hline Tasa de mortalidad perinatal (por 1000 nacimientos) & 2,7 & 1,7 & 0,1 & 1,5 & 6 \\
\hline \multicolumn{6}{|l|}{ Comas } \\
\hline Número de muertes perinatales & 86 & 47 & 3 & 44 & 180 \\
\hline Tasa de mortalidad perinatal (por 1000 nacimientos) & 4,3 & 2,4 & 0,2 & 2,2 & 9,1 \\
\hline Exceso de tasa de mortalidad perinatal ${ }^{* *}$ & 1,6 & 0,7 & 0,1 & 0,7 & 3,1 \\
\hline \multicolumn{6}{|l|}{ Carabayllo } \\
\hline Número de muertes perinatales & 36 & 33 & 1 & 20 & 90 \\
\hline Tasa de mortalidad perinatal (por 1000 nacimientos) & 3,6 & 3,3 & 0,1 & 2 & 9 \\
\hline Exceso de tasa de mortalidad perinatal ${ }^{* *}$ & 0,9 & 1,6 & 0 & 0,5 & 3 \\
\hline
\end{tabular}

* Población tomada como estándar de referencia

** Exceso de tasa de mortalidad perinatal= Tasa de mortalidad perinatal por categoría - tasa de mortalidad perinatal del estándar

Se estimó la tasa de mortalidad perinatal por cada más baja tanto en el 2014 (6,1 por 1000 nacimientos) distrito, donde el distrito de Independencia tuvo la tasa como en el 2015 (5,9 por 1000 nacimientos) (Figura 3).

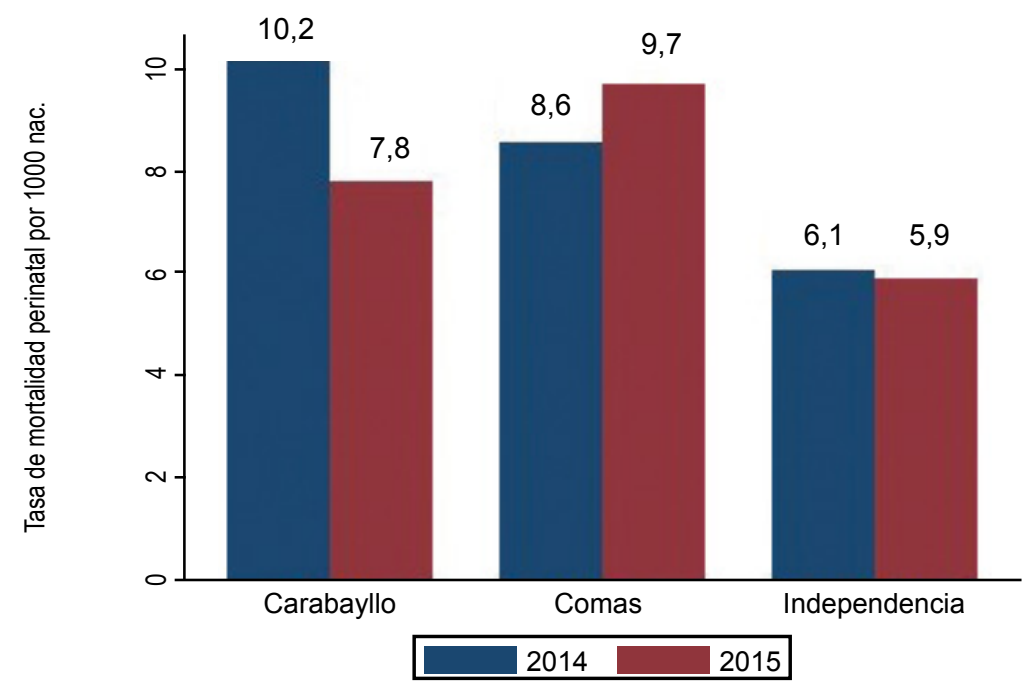

Figura 3. Tasa de mortalidad perinatal de los distritos Carabayllo, Comas e Independencia en los años 2014-2015 


\section{DISCUSIÓN}

En la identificación de la Brecha de Oportunidad con la matriz BABIES se pudieron reconocer las áreas en las que se debería reforzar intervenciones. En Comas encontramos que la Salud materna tuvo el mayor exceso de tasa; esta área incluye las condiciones de la mujer antes del embarazo, momento que se ha descrito como prioritario para fortalecer la planificación familiar, identificar condiciones médicas preexistentes, mejorar la condición nutricional. Además, prevenir, identificar y tratar las infecciones, especialmente, las de trasmisión sexual (ITS) y también prevenir el consumo de sustancias tóxicas como alcohol y drogas ${ }^{(5)}$.

En el distrito de Carabayllo resalta el área de Cuidado durante la etapa prenatal. La intervención a este nivel incluye realizar un control adecuado y de calidad en esta fase para evitar complicaciones, identificar signos de alarma; así también, hacer seguimiento de casos de anemia y del cumplimiento de inmunizaciones, entre otros; lo que permite la reducción de riesgos ${ }^{(5)}$.

El método BABIES ha ayudado a entender la mortalidad perinatal y a monitorizar intervenciones en varias experiencias. En Georgia, se encontró que el área materna requería intervenciones para reducir la tasa de mortalidad (8). Además, en Moldavia se usó esta metodología para monitorizar qué áreas necesitaron mejorar mediante intervenciones ${ }^{(9)}$.

En nuestro país, encontramos un análisis de la mortalidad perinatal en la Dirección de Salud- Cusco (DISA-Cusco) que, aunque se desarrolló a partir de la base de datos de la matriz BABIES, no empleó la metodología BABIES para identificar la Brecha de oportunidad (10). En EE. UU. se emplea una versión adaptada a su realidad denominada Periodos Perinatales de Riesgo o PPOR por sus siglas en inglés ${ }^{(11)}$, la que también ha demostrado ser útil para ver diferencias de mortalidad perinatal de acuerdo a estado socioeconómico o raza ${ }^{(12,13)}$.

Las tasas de mortalidad perinatal encontradas en los distritos de Comas, Carabayllo e Independencia son menores a las registradas a nivel nacional (14). Esta diferencia puede explicarse por las intervenciones que se han realizado en salud materna, neonatal e infantil (15). Además, se debe considerar las ventajas de estar localizados en la capital, donde se encuentra el mayor número de personal de salud, se tiene más información acerca del cuidado a la gestante y un acceso rápido a establecimientos de salud, factores que han sido identificados como claves en la reducción de mortalidad perinatal ${ }^{(16)}$.

Se observó una mayor proporción de casos de mortalidad perinatal alrededor de las 00:00 horas. Esta posible asociación entre la hora de nacimiento y mayor mortalidad perinatal ha sido estudiada desde hace mucho tiempo ${ }^{(17)}$.

Los resultados varían, en algunos estudios se evidencia la relación entre morbilidad y mortalidad con el horario nocturno ${ }^{(18)} y$ en otros no ${ }^{(19)}$. Los que encuentran la relación, la atribuyen a cansancio del personal de salud; sin embargo, recomendamos profundizar la existencia de una posible asociación y evaluar otros factores que podrían estar relacionados.

En relación a las características, se ha visto que existe una proporción del $30 \%$ de los casos que tienen un adecuado peso al nacer, por lo que es necesario identificar otros factores que justifiquen este porcentaje. Por otro lado, alrededor de $67 \%$ tiene bajo peso al nacer, en consistencia con estudios que señalan a esta característica como un factor de riesgo principal asociado a mortalidad perinatal en el Perú ${ }^{(20)}$. Además, la mayoría son prematuros $(71,6 \%)$, un rasgo que también ha sido descrito como relacionado a mayores tasas de mortalidad perinatal ${ }^{(10)}$.

Las características asociadas a la madre fueron las más frecuentes como causa básica de muerte. Sin embargo, en el distrito de Independencia encontramos que la mayoría de casos fueron ocasionados por complicaciones de la placenta, del cordón umbilical y de las membranas. Este perfil de causas de muerte perinatal es parecido, en el caso del distrito de Independencia, con lo reportado en países desarrollados (21) a diferencia de lo encontrado en Carabayllo y Comas que concuerdan con lo hallado en países de bajos y medianos ingresos ${ }^{(22)}$.

En relación a las limitaciones, se trabajó en base al registro secundario de datos de las fichas de vigilancia, lo que tiene una alta variabilidad y posibles casos de muerte perinatal no registradas ${ }^{(23)}$. Además, la misma metodología BABIES solo nos permite evaluar las causas de mortalidad en áreas de intervención de una manera simplificada, por lo que son necesarios estudios posteriores para un análisis específico de la mortalidad (24). Si bien existen métodos que permiten un análisis más detallado, consideramos importante reportar el método de identificación de la Brecha de Oportunidad según el método BABIES porque se puede obtener con pocas variables, es rápido de calcular y resulta útil a nivel local para identificar intervenciones en Salud Pública.

\section{Implicancia en Salud Pública}

Este método permite tomar decisiones a nivel local, tanto por los entes rectores, como el personal de salud en los establecimientos de salud para priorizar acciones como promoción de estilos de vida saludables, campañas de concientización, educación e investigación; y efectivizar el uso de recursos humanos y económicos en las áreas de 
mayor Brecha de Oportunidad, ya sea en salud materna, cuidados durante el embarazo, cuidados durante el parto y cuidados del recién nacido según el método BABIES. Por consiguiente, se podría mejorar los indicadores relacionados a salud materna y neonatal, reducir las tasas de mortalidad perinatal, y obtener un buen desarrollo del binomio madre-niño ${ }^{(5)}$.

\section{Implicancia clínica}

La identificación de la Brecha de Oportunidad permite retroalimentar las prácticas en la actividad asistencial. Estos procedimientos implican realizar una adecuada historia clínica para detectar factores de riesgo, además de signos y síntomas que el diagnóstico de una patología previa al embarazo. Asimismo, se podran identificar patologías fetales, atender el parto en condiciones adecuadas, realizar un óptimo control de puerperio, y dar al recién nacido una atención inmediata que identifique, oportunamente, posibles complicaciones ${ }^{(5,6)}$.

En conclusión, de acuerdo al cálculo de la "Brecha de oportunidades mediante el método BABIES, el área prioritaria para la reducción de mortalidad perinatal en Comas es Salud materna, y en Carabayllo, Cuidados durante el embarazo. Este cálculo permitió comparar tasas en vez de porcentajes relativos como se suele reportar a nivel local. Esta comparación permitió vincular las áreas con un paquete de intervenciones, de acuerdo al método BABIES, para reducir la mortalidad perinatal en las localidades analizadas. Si bien este estudio sólo realizó la identificación de la "Brecha de Oportunidad", consideramos que dado su fácil cálculo y evidencia de su utilidad, se tome en cuenta como un primer análisis de la mortalidad perinatal a nivel local.

\section{Contribución de autores:}

LC y LR participaron en la concepción del artículo, LC, LR, PTL y JCA en la redacción y aprobación de la versión final. LC y LR en la recolección de datos. PTL y JCA realizaron el análisis de datos.

\section{REFERENCIAS BIBLIOGRÁFICAS}

1. World Health Organization. Neonatal and Perinatal Mortality [Internet]. Ginebra: WHO; 2006.

2. Instituto Nacional de Estadística e Informática. Encuesta Demográfica y de Salud Familiar 2017 [Internet]. ENDES 2017. pp. 217-22. Disponible en: https://www.inei.gob.pe/media/ MenuRecursivo/publicaciones_digitales/Est/Lib1525/index. html

3. Instituto Nacional de Salud. Prioridades Nacionales de Investigación 2015-2021 [Internet]. Lima: INEI; 2014. Disponible en: http: //www.portal.ins.gob.pe/es/prioridadesde-investigacion-en-salud

4. Ministerio de Salud. Subsistema Nacional de Vigilancia Epidemiológica Perinatal y Neonatal [Internet]. Lima: MINSA, Direccion General de Epidemiologia, 2009. Disponible en:
http://bvs.minsa.gob.pe/local/minsa/1014_OGE153.pdf

5. Lawn JE, McCarthy BJ, Rae Ross S. The Healthy Newborn: A Newborn Health Management Information System 2. CDC. 2002.

6. Kilonzo A, Kouletio M, McCarthy BJ. Whitehead SJ, Curtis KM. Improving surveillance for maternal and perinatal health in 2 districts of rural Tanzania. Am J Public Health. 2001;91(10):1636-40.

7. Wuhib T, Mccarthy BJ, Chorba TL, Sinitsina TA, Ivasiv I V, Mcnabb SJN, Underestimation of infant mortality rates in one republic of the former Soviet Union. Pediatrics. 2003;111(5 Pt 1):e596-600.

8. Dunlop AL, McCarthy BJ, Freymann GR, Smith CK, Bugg GW, Brann AW. Analysis of feto-infant mortality using the BABIES framework: Georgia 1981-83 through 2001-03. Int J Health Res. 2010;(3):153-63.

9. Stratulat P, Curteanu A, Caraus T, Petrov V, Gardosi J. The experience of the implementation of perinatal audit in Moldova. BJOG. 2014;121(4):167-71.

10. Romero Ramos C, Grajeda Ancca P, Ávila Alatrista J. Análisis de la mortalidad perinatal en Hospitales de la DISA Cusco a partir de la matriz babies 2003. Rev Peru Pediatr. 2004;57(3):28-37

11. Sappenfield WM, Peck MG, Gilbert CS, Haynatzka VR, Bryant T. Perinatal periods of Risk: Analytic preparation and phase 1 analytic methods for investigating feto-infant mortality. Matern Child Health J. 2010;14(6):838-50.

12. Kothari CL, Romph C, Bautista T, Lenz D. Perinatal Periods of Risk Analysis: Disentangling Race and Socioeconomic Status to Inform a Black Infant Mortality Community Action Initiative. Matern Child Health J. 2017;21(Suppl1):49-58.

13. Cai J, Hoff GL, Dew PC, Guillory VJ, Manning J. Perinatal Periods of Risk: Analysis of Fetal-Infant Mortality Rates in Kansas City, Missouri. Matern Child Health J. 2005;9(2):199-205.

14. ENDES. Perú: Encuesta Demográfica y de Salud Familiar 2014. Encuesta Demográfica y Salud Fam; 2015.

15. MINSA. Plan estratégico nacional para la reducción de la mortalidad materna y perinatal 2009-2015. 2009.

16. Merali HS, Lipsitz S, Hevelone N, Gawande. AA, Lashoher A, Agrawal $P$, et al. Audit-identified avoidable factors in maternal and perinatal deaths in low resource settings: a systematic review. BMC Pregnancy Childbirth. 2014; 14:280.

17. Paccaud F, Martin-Béran B, Gutzwiller F. Hour of Birth As a Prognostic Factor for Perinatal Death. Lancet. 1988;331(8581):340-3.

18. Mgaya A, Hinju J, Kidanto $H$. Is time of birth a predictor of adverse perinatal outcome? A hospital-based cross- sectional study in a low-resource setting, Tanzania. BMC Pregnancy Childbirth. 2017;17(1):184.

19. Caughey $A B$, Urato AC, Lee KA, Thiet MP, Washington AE, Laros RK. Time of delivery and neonatal morbidity and mortality. Am J Obstet Gynecol. 2008;199(5):496:e1-5.

20. Ticona RM, Huanco AD. Mortalidad Perinatal Hospitalaria en el Perú : Factores de Riesgo. Rev Chil Obstet Ginecol. 2005; 70:313-7.

21. Flenady V, Middleton P, Smith GC, Duke W, Erwich JJ, Khong TY, et al. Stillbirths: The way forward in high-income countries. Lancet. 2011; 377(9778):1703-17.

22. Aminu M, Unkels R, Mdegela M, Utz B, Adaji S, van den Broek $\mathrm{N}$. Causes of and factors associated with stillbirth in low- and middle-income countries: a systematic literature review. BJOG. 2014;121:141-53. 
23. Espíritu N, Sacieta L, Pantoja L. Discrepancias en el registro de la mortalidad perinatal en Lima y Callao según fuente de información. Rev Perú Med Exp Salud Publica. 2007;24(4):363-9.

24. Nijkamp JW, Sebire NJ, Bouman K, Korteweg FJ, Erwich JJHM, Gordijn SJ. Perinatal death investigations: What is current practice?. Semin Fetal Neonatal Med. 2017;22(3):167-75.

\section{Fuentes de financiamiento:}

Este artículo ha sido financiado por los autores.

Conflictos de interés:

Los autores declaran no tener ningún conflicto de interés.

\section{Correspondencia:}

Paul Jesús Tejada-Llacsa

Dirección: Av. Gonzales Prada 719, Comas. Lima, Perú. Teléfono: 942183586

Correo electrónico: paultejada24@gmail.com

\section{Recibido: 24 de agosto de 2018 .}

Evaluado: 28 de agosto de 2018.

Aprobado: 19 de octubre de 2018

(c) La revista. Publicado por Universidad de San Martín de Porres, Perú. (cc) $\mathbf{B r}$ Licencia de Creative Commons Artículo en acceso abierto bajo términos de Licencia Creative Commons Atribución 4.0 Internacional. (http://creativecommons.org/licenses/by/4.0/)

\section{ORCID iDs}

Luz E. Chan

Lily G. Reyes

Judith Cahuana-Aparco

Paul Jesús Tejada-Llacsa https: / / orcid.org/0000-0001-6148-5011 https://orcid.org/0000-0002-6691-3879 https://orcid.org/0000-0002-8969-3135 https://orcid.org/0000-0003-0830-2786 